\title{
From Seeing to Feeling Sound: A Multimodal Interface for Trans-Domain Mapping of Sound
}

\author{
Rebecca Carter, Thomas McKenzie, Lawrence Sarkar and Kia Ng \\ ICSRiM - University of Leeds \\ School of Electronic and Electrical Engineering, School of Music \& School of Computing, \\ University of Leeds, \\ Leeds LS2 9JT, UK \\ info@icsrim.org.uk,www.icsrim.org.uk
}

\begin{abstract}
Despite its aural focus, sound can encompass both visual and somatic stimulation. This project aims to develop a system to enhance the multimodality of sound using additional visual and haptic feedback techniques. This paper presents a prototype to demonstrate the concept, in the context of technology-enhanced learning for music. With a brief summary of related background literature survey, the paper describes design and development aspects of the system. It discusses software visual interface designs as well as hardware interfaces for vibrotactile feedback to augment an interactive system. The paper concludes with validations and discusses several plausible future directions.
\end{abstract}

Multimodal mapping. Visual. Hearing. Vibrotactile. Sensory experience.

\section{INTRODUCTION}

In this project, we aim to augment sonic experiences through additional sensory modalities, including "seeing" and "hearing". We are interested in exploring multimodal interfaces that allow a user to visualise sound, using graphical representations, whilst feeling it via vibrotactile feedback.

Through transdomain mapping, this project intends to enhance sonic parameters in real-time for several different application scenarios, particularly music pedagogy. To clarify its context, the overall concept is demonstrated using a case study of technology-enhanced learning for singing, using a haptic mapping strategy to allow the user to "feel" if they are "in tune", or not.

\section{BACKGROUND}

This section presents a literature review of related background research including mapping strategies and tools for vocal training purposes.

Ferguson, Vande Moere and Cabrera (2005) developed a visualisation, which provided analytical feedback on a musical performance in real-time, allowing the performer to improve their technique accordingly. Another approach to vocal training is demonstrated by Nanako, Goto and Hiraga (2007). The fundamental frequency of a vocalist was compared, in real-time, to that of a pre-recorded singer and displayed graphically, with a focus on the harmonic content of the voice.

The visual component of this project uses several mapping strategies to translate analysed audio features into visual parameters. There is a range of research into audio-visual relationships that provides the basis for the mapping. Early works include, Simpson, Quinn and Ausubel (1956) which investigated the relationship between colour and pitch in non-synaesthetic children. They identified a association between increasing pitch with yellow, and lower pitches with blue.

In order to convey haptic information to the user, mapping strategies are required to convert sound into physical vibrations. Israr et al. (2012) implement a haptic blur technique that uses amplitude differences between adjacent actuators to create the illusion of a vibration occurring at a position between the actuators. 


\section{DESIGN AND DEVELOPMENT}

This section presents the design and development of a case study based on the multimodal interface concept, to offer visualisation and vibrotactile feedback for singing practice.

The system can be broken down into five different modules; audio input, audio feature extraction, analysis, visualisation, and haptic (see Figure 1).

The vocal input form the microphone is analysed in order to extract audio features. This analysis occurs in a Max MSP Patch where fundamental frequency and amplitude values from the audio input are detected. Data is sent, via OSC (Open Sound Control), to the analysis module for the intonation application where a user-defined reference frequency is compared to the frequency detected.

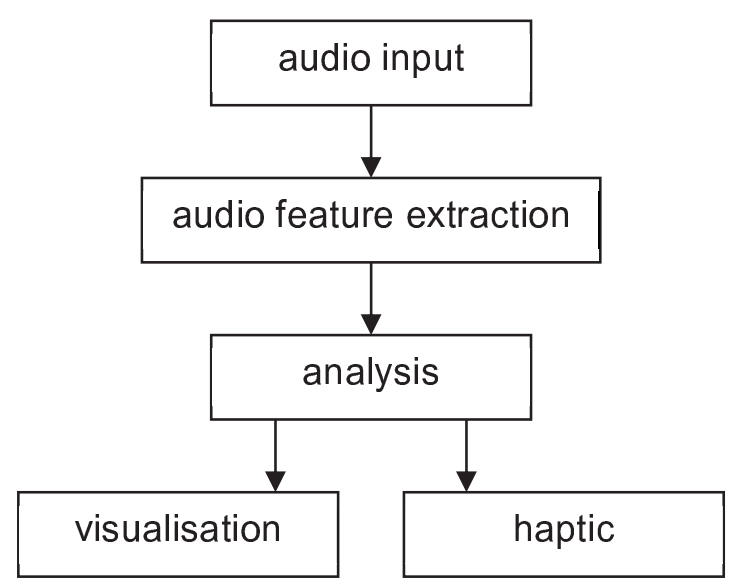

Figure 1: Overall System Architecture

For visual feedback, the interface displays the pitch and intonation of the audio graphically in real-time. The user interface allows the user to select between the different modes and view their performance over time with start and stop buttons.

In order to "feel" how "in" or "out" of tune the vocal line is, this prototype uses an array of haptic actuators on the hand, driven by an Arduino microcontroller. An array of actuators are incorporated into a "vibrotactile feedback interface" (see Figure 2) in a straight line of equal spacing to align from the tip of the middle finger to the base of the hand, relative to the average hand size. The user puts his/her hand on top of the interface and it moulds accordingly. The interface consists of a plastic bag filled with a gel. A range of substances with different viscosities, including rice and custard, have been tested and toothpaste proved to be most effective, as it did not audibly amplify the vibrations and moulded to the shape of the hand.

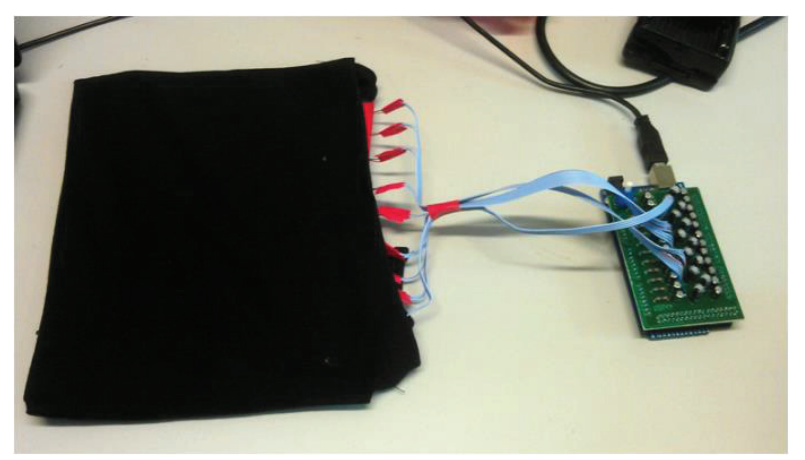

Figure 2: Vibrotactile Feedback Interface

For this specific case study, pitch and intonation interfaces have been designed. The pitch interface allows the user to see and feel the pitch of their vocal performance over time (see Figure 3). A line is drawn on the visual interface for each note sung, with pitch indicated by the vertical position of the line on a logarithmic scale. The amplitude of the audio determines the opacity of the note line, increasing with amplitude. Similar to the visualisation, the haptic interface maps pitch to relative position on the actuator array, and amplitude to vibration strength.

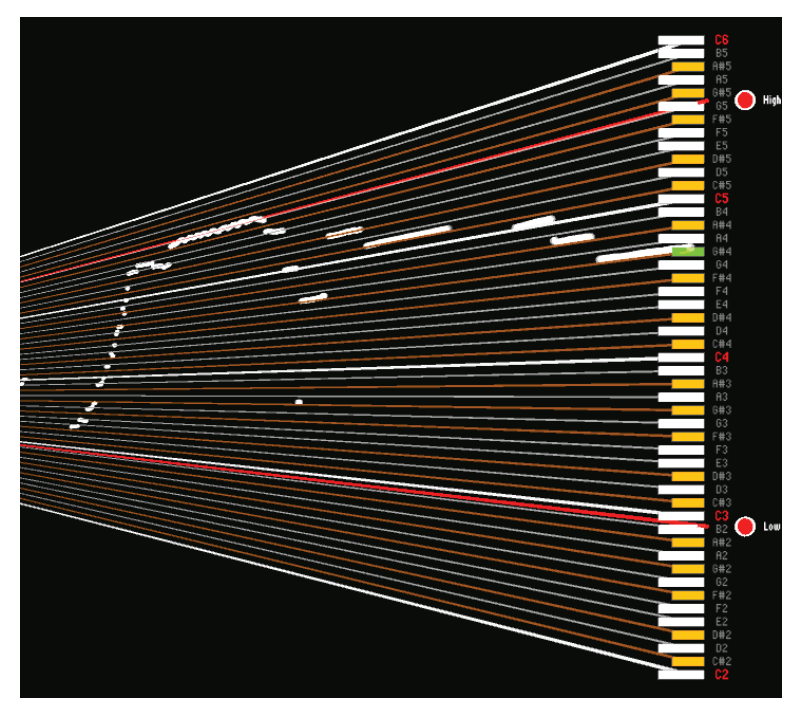

Figure 3: Pitch Visualisation

The intonation mode allows the user to "see" and "feel" tuning, relative to the reference frequency set at the start of the performance (see Figure 4). The position of the bar changes depending on accuracy. The distance of the bar from the centre position is determined by the number of cents away from the intended pitch. It moves up when a sharper note is detected. The colour of the bar also becomes increasingly more yellow to indicate 
sharper frequency. The bar moves down for flatter input and the colour of the bar becomes increasingly blue to indicate degree of flatness (Simpson, Quinn \& Ausubel, 1956).

The vibrotactile interface does not produce any vibration if the audio is in tune. If the audio is out of tune, a vibration occurs on the hand at a position that correlates with the visualisation. Notes that are further out of tune have a greater strength of vibration.
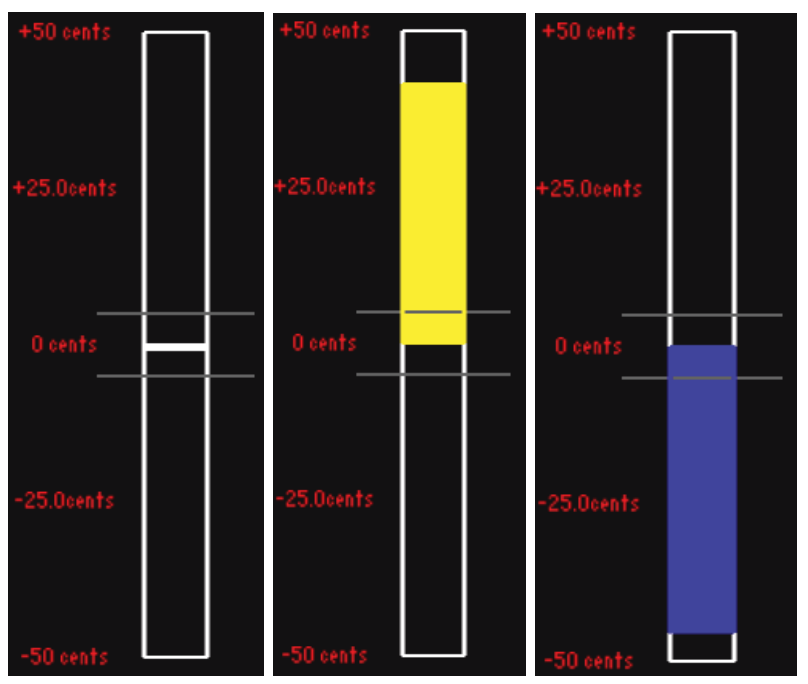

Figure 4: Intonation Visualisation Displaying In Tune (Left), Sharp (Middle) and Flat (Right)

\section{VALIDATIONS}

To ascertain the effectiveness of the prototype, a qualitative validation was carried out with 30 subjects. A demonstration of the pitch and intonation interfaces was given before a user test. After the test, a questionnaire was completed.

Feedback was generally positive, with subjects indicating that the pitch analysis interface would be most useful at a beginner level and intonation at more advanced levels.

In respect to the vibrotactile feedback, half of the subjects identified that they did not feel the vibrations evenly. This exemplifies the individualistic nature of human beings. Not only do we hear and see differently, we also feel things differently, highlighting a need for individual configurations of the vibrotactile system.

\section{SUMMARY AND FURTHER WORK}

This paper has introduced a multimodal project for real-time haptic and visual feedback of audio in the context of a technology-enhanced learning tool for vocalists. The application scenario presented in this paper aims to increase understanding of music and improve a vocal performer's technique through engaging the senses of sight and touch. This paper has considered existing, related systems and visual and haptic mapping strategies. Aspects of the design and development of the system have been described, followed by a brief description of validation conducted on the prototype.

The concept of this project; representing aspects of sound with different senses, could be extended beyond musical applications. Wearable haptic devices have recently entered the consumer market with devices such as the 3rd Space Vest (http://tngames.com/), designed to give force feedback in video games and TapTap, a scarf designed for emotional therapy (Bonanni, Lieberman \& Vaucelle, 2006). With miniaturisation and increasing processing power, it is feasible that the system presented in this project could be embedded into wearable devices that can continuously analyse and translate surrounding sound.

\section{REFERENCES}

Bonanni, L., Lieberman, J., Vaucelle, C., Zuckerman, O. (2006), 'TapTap: A Haptic Wearable for Asynchronous Distributed Touch Therapy' CHI 2006 Montréal, Quebec, Canada, April 22-27.

Ferguson, S., VandeMoere, A., Cabrera, D. (2005), 'Seeing sound: real-time sound visualisation in visual feedback loops used for training musicians' Information Visualisation, 2005 Proceedings.

Israr, A., Kim, S.C., Poupyrev, I., Stec, J. (2012), 'Surround Haptics: Tactile Feedback for Immersive Gaming Experiences' CHI 2012, Austin, Texas, May 10-15.

Nakano, T., Goto, M., Hiraga, Y. (2007), 'MiruSinger: A singing skill visualisation interface using real-time feedback and music $C D$ recordings as referential data' Ninth IEEE International Symposium on Multimedia 2007 - Workshops.

Simpson, R.H., Quinn, M., Ausubel, D.P. (1956), 'Synesthesia in Children: Association of Colors with Pure Tone Frequencies' Journal of Genetic Psychology, Vol. 89, pp. 95-103. 